\title{
Correlation between Left Ventricular Hypertrophy in Electrocardiogram and Hypertension in Physical Examination Population
}

\author{
LI LI CHEN, LI QING CHEN AND NING MA*
}

Department of Electrocardiogram room, The Central Hospital of Shengli Oil Field, Dongying, Shandong, 257034, China

Chen et al.: Correlation between Left Ventricular Hypertrophy in Electrocardiogram and Hypertension

To explore the correlation between left ventricular hypertrophy in electrocardiogram and hypertension in the population underwent physical examination. A total of 96 patients who underwent physical examination in our hospital from February 2019 to February 2020 were selected as the research subjects. 48 patients with normal electrocardiogram were selected as the control group and 48 patients with left ventricular hypertrophy showed by electrocardiogram were selected as the observation group. $24 \mathrm{~h}$ systolic blood pressure, $24 \mathrm{~h}$ diastolic blood pressure, nocturnal systolic blood pressure, nocturnal diastolic blood pressure, the standard deviation of $24 \mathrm{~h}$ systolic blood pressure, the standard deviation of nocturnal systolic blood pressure and the standard deviation of nocturnal diastolic blood pressure were compared between the two groups. Compared with the control group, there were 20 patients with hypertension in the observation group and the detection rate was $41.67 \%$. There were 3 patients with hypertension in the control group and the detection rate was $6.25 \%$. The detection rate of hypertension in the observation group was significantly higher than that in the control group $(\mathbf{p}<0.05)$. Compared with the control group, $24 \mathrm{~h}$ systolic blood pressure, $24 \mathrm{~h}$ diastolic blood pressure, nocturnal systolic blood pressure, nocturnal diastolic blood pressure, the standard deviation of $24 \mathrm{~h}$ systolic blood pressure, the standard deviation of nocturnal systolic blood pressure and the standard deviation of nocturnal diastolic blood pressure in observation group were significantly increased $(\mathrm{p}<\mathbf{0 . 0 5})$. The incidence of myocardial infarction and sudden death in observation group was significantly higher than that in control group $(p<0.05)$. The left ventricular hypertrophy shown by electrocardiogram is of great value to the detection rate of hypertension in physical examination. It can evaluate the blood pressure status of patients and has good clinical application value.

Key words: Electrocardiogram, left ventricular hypertrophy, hypertension, electrocardiogram

With the continuous advance of social economy and people's living standards, the incidence of some chronic diseases is on the rise. Essential hypertension ${ }^{[1,2]}$ is a common chronic disease, which also serves as the most common cardiovascular disease in society at present. Relevant data showed that, with the aggravation of aging in recent years, about $1 / 3^{\text {rd }}$ adults suffered from hypertension and nearly 750000 people died from cardiovascular and cerebrovascular accidents caused by hypertension every year. Essential hypertension is a syndrome with increased systemic arterial pressure as the main clinical symptom. Its clinical symptoms are not obvious in the early stage of the disease and it is easy to be ignored by patients, with a relatively low detection rate $^{[3]}$. Clinical studies informed that ${ }^{[4]}$, if patients are not treated in time, they are prone to serious diseases such as atherosclerosis, or even cerebral hemorrhage, which seriously affect the prognosis and quality of life of patients affected. A large number of studies ${ }^{[5,6]}$ have shown that left ventricular hypertrophy is one of the common target organ injury in essential hypertension, which is an adaptive response to the long-term increase of hemodynamic load. Hypertensive left ventricular hypertrophy is also a major independent risk factor for all cardiovascular complications. The emergence of left ventricular hypertrophy will inevitably exert an impact on ventricular depolarization and negative pole, so the early accurate measurement of left ventricular wall thickness is of great value in the prevention of hypertension and coronary heart disease. In clinic,

*Address for correspondence

E-mail: zxyyxdtmn@163.com 
the electrocardiogram (ECG), echocardiography (UCG) and other non-invasive methods are generally used to diagnose the occurrence of left ventricular hypertrophy, among which ECG has the advantages of simplicity, economy and good repeatability ${ }^{[7]}$. Therefore, in this paper, the relationship between the left ventricular hypertrophy and hypertension in the physical examination population was studied, in order to provide a reference for the prevention and treatment of clinical hypertension, which was reported as follows.

\section{MATERIALS AND METHODS}

\section{General data:}

A total of 96 physical examiners who underwent physical examination in our hospital from February 2019 to February 2020 were selected as the research objects, including 47 males and 49 females, aged from 30 to $70 \mathrm{y}$ old, with an average age of $50.48 \pm 3.06 \mathrm{y}$ old. Based on results of physical examination, 48 cases with normal electrocardiogram were taken as the control group and 48 cases with left ventricular hypertrophy showed by the electrocardiogram were taken as the observation group. In the control group, there were 23 males and 25 females, with an average age of $51.03 \pm 3.26 \mathrm{y}$ old. There were 24 males and 24 females in the observation group, with an average age of $50.06 \pm 3.29 \mathrm{y}$ old. All the subjects and their families knew about this study and had signed the informed consent.

\section{Inclusion criteria and exclusion criteria:}

Inclusion criteria: Patients with complete clinical data; patients who were able to complete this researcher; the observation group was clinically diagnosed as essential hypertension, which conformed to the standard of "Guidelines for Prevention and Treatment of Hypertension in China"[8]; patients with no family history of mental illness.

Exclusion criteria: Patients with severe deficiency of important organs such as liver and kidney; Lactating or pregnant women; patients complicated with serious autoimmune diseases; patients complicated with stroke; patients who were diagnosed as the secondary hypertension by thyroid function, catecholamine, renin-angiotensin, aldosterone level, ultrasound of both kidneys and renal arteries and computed tomography (CT) examination of bilateral adrenal glands.

\section{Detection methods:}

Left ventricular hypertrophy in ECG: All subjects underwent ECG. First, the subjects were placed in a supine position and examined by 12-lead synchronous ECG. The left ventricular hypertrophy of all subjects was evaluated by Romhilt Esters integration method and the main contents of Romhilt Esters integration method were as follows: any one of the following QRS amplitudes was scored as 3 points, including: $\mathrm{R}$ or $\mathrm{S}$ wave $\geq 20 \mathrm{~mm}$; in V1 or V2, S wave $\geq 30 \mathrm{~mm}$; in V5 or $\mathrm{V} 6, \mathrm{~S}$ wave $\geq 30 \mathrm{~mm}$, in $\mathrm{V} 1$ or $\mathrm{V} 2, \mathrm{~S}$ wave $\geq 30 \mathrm{~mm}$. Changes in ST-T segment: no digitalis was used for 3 points, digitalis for 1 point and left axis deviation $\geq-30^{\circ}$ for 2 points. Left atrial complex overweight: 3 points at $\mathrm{V} 1$ terminal negative $\mathrm{P}$ wave depth $\geq 1 \mathrm{~mm}$, or time limit $\geq 0.04 \mathrm{~s}, 1$ point at $\mathrm{QRS}$ complex time $\geq 0.09 \mathrm{~s}, 1$ point at V5-6 VAT $=0.05 \mathrm{~s}$ and left ventricular hypertrophy was diagnosed when patient score $\geq 5$ points.

The detection method of blood pressure: All subjects were detected by automatic non-invasive blood pressure monitor and cuffs were tied to the left arm of the subjects. $24 \mathrm{~h}$ systolic blood pressure ( $24 \mathrm{~h} \mathrm{SBP}$ ), diastolic blood pressure (DBP) and standard deviation systolic blood pressure (SBP SD) were detected. At the same time, the nocturnal systolic blood pressure (NSBP), nocturnal diastolic blood pressure (NDBP) and standard deviation of nocturnal systolic blood pressure (NSBP SD) of the two groups were falsely detected. During the testing period, the examinee should be advised to avoid or minimize strenuous exercise and mood fluctuation as far as possible that may affect the test results.

\section{Observation indexes:}

The detection rate of hypertension-Statistics were carried out on patients with hypertension in the two groups of research objects and the detection rate was calculated and compared; The ambulatory blood pressure-24 h SBP, $24 \mathrm{~h}$ DBP, NSBP, NDBP, $24 \mathrm{~h}$ SBP SD, the NSBP SD and NDBP SD were compared between the two groups; The occurrence of cardiovascular events-hypertensive patients with or without ventricular hypertrophy were followed up for 2 $y$ and the number of cases of myocardial infarction and sudden death was counted and compared.

\section{Statistical analysis:}

The data were analyzed by statistical package for the social sciences (SPSS) 22.0 statistical software. In detail, the measurement data in accordance with the normal distribution were expressed as the mean \pm standard deviation $(\overline{\mathrm{x}} \pm \mathrm{s})$. The comparison between groups was conducted by t-test. The counting data were expressed as the number of cases (n) or percentage (\%) and the 
data was tested by $\chi^{2}$ test. $\mathrm{p}<0.05$ indicates that the difference is statistically significant.

\section{RESULTS AND DISCUSSION}

There were no significant differences in the gender, the mean age and the course of disease and body mass index (BMI) between the two groups $(\mathrm{p}>0.05)$, as shown in Table 1.

In the observation group, 20 patients with hypertension were detected and the detection rate was $41.67 \%$. In the control group, 3 patients with hypertension were detected, with a detection rate of $6.25 \%$. The detection rate in the observation group was significantly higher than that in the control group, with statistically significant difference $(\mathrm{p}<0.05)$. As shown in Table 2 , fig. 1 and fig. 2.

The $24 \mathrm{~h}$ SBP, $24 \mathrm{~h}$ DBP, NSBP, NDBP, $24 \mathrm{~h}$ SBP SD, NSBP SD and NDBP SD were significantly higher in the observation group than those in the control group. And the differences were statistically significant $(\mathrm{p}<0.05)$, see Table 3 .

The incidence of myocardial infarction and sudden death was significantly higher in the observation group than that in the control group and the difference was statistically significant $(\mathrm{p}<0.05)$, as shown in Table 4.

TABLE 1: COMPARISON OF GENERAL DATA BETWEEN TWO GROUPS OF SUBJECTS

\begin{tabular}{|c|c|c|c|c|c|}
\hline Group & & $\begin{array}{l}\text { Observation group } \\
(n=48)\end{array}$ & $\begin{array}{l}\text { Control group } \\
(n=48)\end{array}$ & Statistical value & $p$ value \\
\hline \multirow{2}{*}{ Gender (case) } & Male & 24 & 23 & \multirow{2}{*}{5.267} & \multirow{2}{*}{0.492} \\
\hline & Female & 24 & 25 & & \\
\hline Mean age (y old) & & $50.06 \pm 3.29$ & $51.03 \pm 3.26$ & 4.269 & 1.003 \\
\hline BMI $\left(\mathrm{kg} / \mathrm{m}^{2}\right)$ & & $22.15 \pm 3.25$ & $22.01 \pm 3.57$ & 4.198 & 0.129 \\
\hline
\end{tabular}

TABLE 2: COMPARISON OF THE DETECTION RATE OF HYPERTENSION BETWEEN THE TWO GROUPS [n (\%)]

\begin{tabular}{lcccc}
\hline Group & $\begin{array}{c}\text { Observation group } \\
(n=48)\end{array}$ & Control group $(n=48)$ & Statistical value & p value \\
\hline Hypertension & $20(41.67)$ & $3(6.25)$ & 4.198 & 0.129 \\
\hline
\end{tabular}

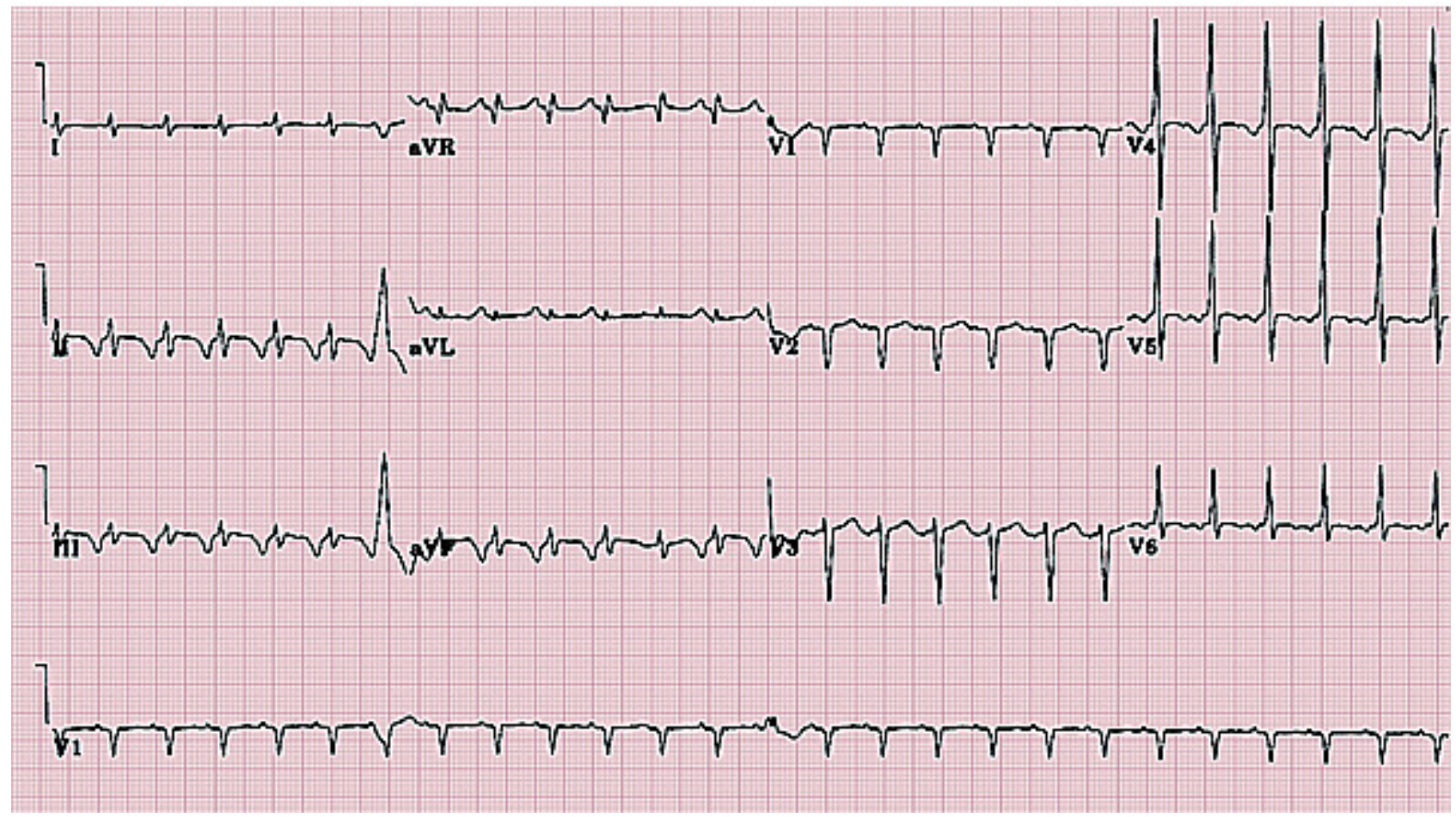

Fig. 1: Electrocardiographic findings of hypertensive patients without left ventricular hypertrophy 


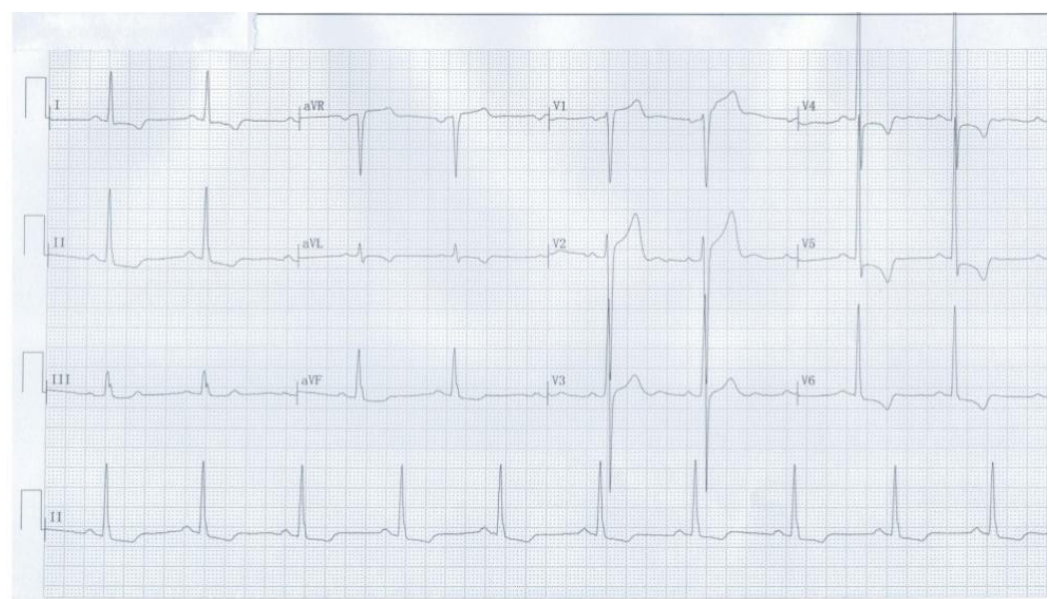

Fig. 2: Electrocardiographic findings of hypertensive patients with left ventricular hypertrophy

TABLE 3: COMPARISON OF DYNAMIC BLOOD PRESSURE BETWEEN THE TWO GROUPS ( $\bar{x} \pm s$ )

\begin{tabular}{lcccc}
\hline Group & $\begin{array}{c}\text { Observation group } \\
(\mathbf{n}=\mathbf{4 8})\end{array}$ & Control group $(\mathbf{n}=\mathbf{4 8})$ & T value & p value \\
\hline 24 h SBP & $130.48 \pm 15.24$ & $114.25 \pm 12.02$ & 6.025 & 0.001 \\
24 h DBP & $78.59 \pm 10.03$ & $70.01 \pm 8.59$ & 5.263 & 0.005 \\
NSBP & $124.03 \pm 15.20$ & $110.03 \pm 10.06$ & 5.987 & 0.016 \\
NDBP & $73.49 \pm 10.05$ & $63.20 \pm 8.29$ & 5.219 & 0.002 \\
24 h SBP SD & $13.29 \pm 4.15$ & $10.06 \pm 3.21$ & 6.007 & 0.003 \\
NSBP SD & $14.62 \pm 4.19$ & $10.27 \pm 2.95$ & 5.221 & 0.008 \\
NDBP SD & $10.26 \pm 2.57$ & $7.06 \pm 2.18$ & 5.264 & 0.005 \\
\hline
\end{tabular}

TABLE 4: COMPARISON OF INCIDENCE OF CARDIOVASCULAR EVENTS BETWEEN THE TWO GROUPS [n (\%)]

\begin{tabular}{lcccc}
\hline Group & $\begin{array}{c}\text { Observation group } \\
(\mathrm{n}=48)\end{array}$ & Control group $(\mathrm{n}=48)$ & $\chi^{2}$ value & p value \\
\hline Myocardial infarction & $5(10.41)$ & $1(2.08)$ & 5.269 & 0.002 \\
Sudden death & $6(12.50)$ & $1(2.08)$ & 6.007 & 0.004 \\
$\begin{array}{l}\text { Total number of } \\
\text { occurrences }\end{array}$ & $11(22.92)$ & $2(4.17)$ & 5.201 & 0.005 \\
\hline
\end{tabular}

Essential hypertension is a chronic disease induced by genetic, environmental and other factors, which requires patients to take medicine for a long time. If blood pressure is not controlled in time, patients will undergo abnormal changes in the heart and vascular function and structure, which will seriously affect the life safety and quality of life of patients ${ }^{[9]}$. A line of evidence indicated that ${ }^{[10]}$ patients with hypertension showed no specific clinical manifestations in the early stage and the rising blood pressure may increase their mental load, resulting in left ventricular hypertrophy, thus leading to cardiac enlargement and even heart failure. Even though there are multiple factors resulting in left ventricular hypertrophy, including cardiomyopathy, weight loss and myocardial amyloidosis, the most common factor is hypertension. Left ventricular hypertrophy is the earliest target organ injury caused by hypertension. With the increase of blood pressure, the left ventricular load increases, resulting in thickening of ventricular wall and increase of tension. In order to maintain the previous blood pumping ability, left ventricular hypertrophy gradually appears ${ }^{[11]}$. Therefore, accurate measurement of blood pressure can help comprehensively understand the characteristics of blood pressure changes in patients with hypertension complicated with left ventricular hypertrophy, which plays a guiding role in the prevention and treatment of clinical hypertension. Early clinical diagnosis can effectively improve the therapeutic effect of patients with hypertension and control the level of blood pressure. In the detection of blood pressure, 24 $\mathrm{h}$ ambulatory blood pressure monitoring can help to grasp the blood pressure of patients in real time and can eliminate the errors caused by personal measurement, which will not be restricted by the physical activity and 
sleep of patients. It can reflect the changes of patients' blood pressure in a day ${ }^{[12]}$. However, clinical studies have shown that the cost of this method is high and its clinical adaptability has some limitations. Therefore, the detection of ambulatory blood pressure is mostly used in the follow-up treatment of patients with hypertension. Electrocardiogram is a common physical examination item in clinic. Compared with ambulatory blood pressure monitoring, it has the advantages of simplicity, rapidness and low price, which can be accepted by more patients and left ventricular hypertrophy can be better judged by $\mathrm{ECG}^{[13]}$.

Previous studies have found that hypertension is closely related to the occurrence of left ventricular hypertrophy. Zhiling and other scholars ${ }^{[14]}$ found that the probability of hypertension in patients with left ventricular hypertrophy was significantly higher than that in normal people. Yun et al. ${ }^{[15]}$ pointed out that the diagnosis of left ventricular hypertrophy by electrocardiogram plays an early warning role in the occurrence of hypertension. Cicero et al. ${ }^{[16]}$ found that left ventricular hypertrophy has become an independent risk factor for cardiovascular disease morbidity and mortality through the detection of $24 \mathrm{~h}$ ambulatory blood pressure. In the above study, it was found that there were 20 patients with hypertension in the observation group and the detection rate of hypertension was 41.67 $\%$, while only 3 patients in the control group developed hypertension and the detection rate was only $6.25 \%$. The detection rate of hypertension in the observation group was significantly higher than that in the control group. To some extent, this showed that the diagnosis of left ventricle by ECG can predict the occurrence of hypertension. This was consistent with the research results of many scholars mentioned above. However, according to previous clinical studies, the risk factors of left ventricular patients mainly include the obesity, smoking, diabetes and other factors, which can cause target organ damage. Therefore, it is necessary to understand the clinical data of patients in the evaluation of left ventricular hypertrophy.

Liu Yang and other scholars ${ }^{[17]}$ found that in the ECG, the blood pressure indexes of patients with ventricular hypertrophywere significantly higher than those of normal people. Yanan et al. ${ }^{[18]}$ pointed out that compared with the normal level, the blood pressure of patients with left ventricular hypertrophy increased significantly. In the above study, it was found that 24 $h$ SBP, $24 \mathrm{~h}$ DBP, NSBP, $24 \mathrm{~h}$ DBP SD, NSBP SD and NDBP SD in the observation group were significantly higher than those in the control group. This result fully confirmed that the left ventricular hypertrophy can be used as an early warning signal for hypertension. The above studies demonstrated that the increase in nocturnal blood pressure was more significant, which may be due to nocturnal hypertension, thus indirectly increasing the burden of the vascular system, further promoting the synthesis of cardiac fibrin and changes in myocardial hypertrophy, which will eventually promote the occurrence of cardiovascular events. This phenomenon was also found in the above research. In addition, the SD of SBP and DBP in the observation group was significantly higher than the normal value, which indirectly increased the risk of cardiovascular disease and target organ injury, which was in line with the conclusion of Kim et al. ${ }^{[19]}$. The occult hypertension is an easily missed disease and clinical studies have shown that occult hypertension can also increase the incidence of cardiovascular disease and target organ damage. It is the main predictor of cardiovascular disease and death. Therefore, the clinical examination of ECG in patients with ventricular hypertrophy ${ }^{[20]}$ is of great significance to the life and prognosis of the patients. In the above study, it was also found that the incidence of myocardial infarction and sudden death complications in the observation group was significantly higher than that in the control group, suggesting that hypertension can predict the occurrence of cardiovascular events. This was consistent with the results of Chen Jixiong and other scholars.

To sum up, the left ventricular hypertrophy in ECG plays a certain role in predicting the occurrence of hypertension, which is of great significance to the prognosis and life safety of patients and provides more basis for later clinical diagnosis and treatment. Thus, it has high clinical application value.

\section{Acknowledgements:}

None.

\section{Conflicts of interest:}

The authors report no conflicts of interest.

\section{REFERENCES}

1. Flynn JT. High blood pressure in the young: why should we care? Acta Paediatr 2018;107(1):14-9.

2. Carey RM, Whelton PK. 2017 ACC/AHA Hypertension Guideline Writing Committee. Prevention, Detection, Evaluation, and Management of High Blood Pressure in Adults: Synopsis of the 2017 American College of Cardiology/ American Heart Association Hypertension Guideline. Ann Intern Med 2018;038(005):479-482. 
3. Barrera L. High blood pressure prevention and control: From evidence to action. Colomb Med 2018;49(2):137-8.

4. Brady TM, Stefani GA, Simonetti GD. Management of high blood pressure in children: similarities and differences between US and European guidelines. Pediatr Nephrol 2019;34(3):40512.

5. Luo D, Cheng $\mathrm{Y}$, Zhang $\mathrm{H}, \mathrm{Ba} \mathrm{M}$, Chen $\mathrm{P}$, Li $\mathrm{H}$, et al. Association between high blood pressure and long term cardiovascular events in young adults: systematic review and meta-analysis. BMJ 2020;370.

6. Baker-Smith CM, Flinn SK, Flynn JT, Kaelber DC, Blowey $\mathrm{D}$, Carroll AE, et al. Diagnosis, evaluation and management of high blood pressure in children and adolescents. Pediatrics 2018;142(3).

7. Wang J, Qiu D, Tu X. Correlation analysis between blood pressure variability and left ventricular mass index in patients with essential hypertension. Chin J Aged Cardiovasc Cerebrovasc Dis 2020;7(9):4517-22.

8. 2018 Chinese Guidelines for Prevention and Treatment of Hypertension - A report of the Revision Committee of Chinese Guidelines for Prevention and Treatment of Hypertension. J Geriatr Cardiol 2019;16(3):182-241.

9. Hinton TC, Adams ZH, Baker RP, Hope KA, Paton JF, Hart $\mathrm{EC}$, et al. Investigation and treatment of high blood pressure in young people: too much medicine or appropriate risk reduction? Hypertension 2020;75(1):16-22.

10. Muntner P, Carey RM, Gidding S, Jones DW, Taler SJ, Wright Jr JT, et al. Potential US population impact of the 2017 ACC/AHA high blood pressure guideline. Circulation 2018;137(2):109-18.

11. Yang Z, Bai Y, Wang S. Correlation between serum thiocerebroside and QT dispersion and NT-proBNP levels in patients with essential hypertension and left ventricular hypertrophy. J Mod Integr Tradit Chin West Med 2020;8(8):2830.

12. Pazoki R, Dehghan A, Evangelou E, Warren H, Gao H, Caulfield $\mathrm{M}$, et al. Genetic predisposition to high blood pressure and lifestyle factors: associations with midlife blood pressure levels and cardiovascular events. Circulation 2018;137(7):653-61.
13. Miu Y, Xu M, Zhu Y. Diagnostic value of improved Cornell voltage in hypertensive left ventricular hypertrophy. Tianjin Med 2020;8(10)18-23.

14. Ma Z, Zhao Z, Ye C. Differentiation of left ventricular hypertrophy and hypertrophic cardiomyopathy caused by hypertension by surface electrocardiogram and echocardiography. J Heart 2019;8(9):526-39.

15. Zhu Y, Yang P, Ge Z. The value of electrocardiogram S D wave in the diagnosis of left ventricular hypertrophy in patients with hypertension. Chin J Arterioscler 2020;12(7):174-84.

16. Cicero AF, Grassi D, Tocci G, Galletti F, Borghi C, Ferri C. Nutrients and nutraceuticals for the management of high normal blood pressure: an evidence-based consensus document. High Blood Press Cardiovasc Prev 2019;26(1):9-25.

17. Liu Y, Shi L, Lin Y, Zheng T, Li XH, Liu YY, et al. Relationship between blood pressure variability and target organ damage in children with essential hypertension. Chin J Pediatr 2019;57(2):93-7.

18. Zhang $\mathrm{Y}, \mathrm{Xu}$ J, Yao L. Application value of ambulatory electrocardiogram and ambulatory blood pressure monitoring in clinical patients with renal hypertension. Chin Med 2019;16(31):46-51.

19. Kim S, Goel R, Kumar A, Qi Y, Lobaton G, Hosaka K, et al. Imbalance of gut microbiome and intestinal epithelial barrier dysfunction in patients with high blood pressure. Clin Sci 2018;132(6):701-18.

20. Chen J, Huang X, Wang P. Clinical study on sympathetic nerve activity in elderly patients with hypertension and left ventricular hypertrophy. J Cardiovasc Rehabil Med 2019;28(12):2211-3.

This is an open access article distributed under the terms of the Creative Commons Attribution-NonCommercial-ShareAlike 3.0 License, which allows others to remix, tweak, and build upon the work non-commercially, as long as the author is credited and the new creations are licensed under the identical terms

This article was originally published in a special issue,
"Therapeutic Perspectives in Biomedical Research and Pharma-
ceutical Sciences and their Nursing Methods"
Indian J Pharm Sci 2021:83(4)Spl issue "56-61"

